\title{
A SIXTH-ORDER NONLINEAR PARABOLIC EQUATION FOR QUANTUM SYSTEMS*
}

\author{
ANSGAR JÜNGEL ${ }^{\dagger}$ AND JOSIPA-PINA MILIŠIĆ $\ddagger$
}

\begin{abstract}
The global-in-time existence of weak nonnegative solutions to a sixth-order nonlinear parabolic equation in one space dimension with periodic boundary conditions is proved. The equation arises from an approximation of the quantum drift-diffusion model for semiconductors and describes the evolution of the electron density in the semiconductor crystal. The existence result is based on two techniques. First, the equation is reformulated in terms of exponential and power variables, which allows for the proof of nonnegativity of solutions. The existence of solutions to an approximate equation is shown by fixed-point arguments. Second, a priori bounds uniformly in the approximation parameters are derived from the algorithmic entropy construction method which translates systematic integration by parts into polynomial decision problems. The a priori estimates are employed to show the exponential time decay of the solution to the constant steady state in the $L^{1}$ norm with an explicit decay rate. Furthermore, some numerical examples are presented.
\end{abstract}

Key words. Sixth-order parabolic equation, quantum semiconductors, global existence of solutions, algorithmic entropy construction, convergence of transport plans, long-time behavior of the solutions.

AMS subject classifications. 35K30, 35K55, 35Q40, 35B40.

1. Introduction. We prove the global-in-time existence of weak nonnegative solutions to the following initial-value problem on the one-dimensional torus $\mathbb{T}$ with unit length:

$$
\begin{aligned}
& n_{t}=L[n]:=\left[n\left(\frac{1}{n}\left(n(\log n)_{x x}\right)_{x x}+\frac{1}{2}\left((\log n)_{x x}\right)^{2}\right)_{x}\right]_{x}, \quad x \in \mathbb{T}, t>0, \\
& n(x, 0)=n_{0}(x), \quad x \in \mathbb{T} .
\end{aligned}
$$

Equation (1.1) originates from a generalized quantum drift-diffusion model for semiconductors, derived from a Wigner-BGK model using a moment method [10]. It is obtained from the $O\left(\delta^{6}\right)$ approximation of the quantum drift-diffusion model, where $\delta>0$ is the scaled Planck constant; see the appendix of [15]. The variable $n(x, t)$ represents the electron density in the semiconductor crystal and should be a nonnegative quantity. Expanding the derivatives, the above equation can be written as

$$
\begin{aligned}
n_{t}= & \left(n^{(\mathrm{v})}-3 n^{-1} n^{(\mathrm{iv})} n_{x}-5 n^{-1} n_{x x x} n_{x x}+8 n^{-2} n_{x x x} n_{x}^{2}-18 n^{-3} n_{x x} n_{x}^{3}\right. \\
& \left.+6 n^{-4} n_{x}^{5}+11 n^{-2} n_{x x}^{2} n_{x}\right)_{x},
\end{aligned}
$$

where $n^{(\mathrm{iv})}$ and $n^{(\mathrm{v})}$ denote the fourth- and fifth-order derivatives, respectively, and $n_{x}^{a}$ abbreviates $\left(n_{x}\right)^{a}$ for $a \in \mathbb{N}$, showing that (1.1) is of parabolic type.

${ }^{*}$ The first author acknowledges partial support from the Austrian Science Fund (FWF), grant P20214 and WK "Differential Equations", and from the German Science Foundation (DFG), grant JU 359/7. Both authors have been supported by the Austrian-Croatian Project of the Austrian Exchange Service (ÖAD) and the Ministry of Science, Education, and Sports of the Republic of Croatia (MZOS). This research is part of the ESF program "Global and geometrical aspects of nonlinear partial differential equations (GLOBAL)". The authors thank the anonymous referees for comments helping to improve the paper. The first author thanks G. Savaré for very useful discussions.

${ }^{\dagger}$ Institute for Analysis and Scientific Computing, Vienna University of Technology, Wiedner Hauptstr. 8-10, 1040 Wien, Austria (juengel@anum.tuwien.ac.at)

$\ddagger$ Department of Applied Mathematics, Faculty of Electrical Engineering and Computing, University of Zagreb, Unska 3, 10000 Zagreb, Croatia (pina@webmail.fer.hr) 
The original model of [15], without electric field, reads as

$$
n_{t}=n_{x x}-\frac{\delta^{2}}{2}\left(n(\log n)_{x x}\right)_{x x}+\frac{\delta^{4}}{10} L[n] .
$$

We consider here only the main part of the model since the treatment of the secondand fourth-order expressions is by now well understood and much easier than the sixth-order term. For an existence proof of the fourth-order equation (without the $L[n]$ term), we refer to [18]. We recover (1.1) after neglecting the second- and fourthorder terms and setting $\delta^{4}=10$.

It is possible to derive an $O\left(\delta^{2 m}\right)$ approximation of the above mentioned WignerBGK model, leading to an evolution equation of order $2 m$. However, its nonlinear structure is highly complicated and, moreover, a general formulation for all $m$ seems to be not available. Therefore, we analyze in this paper the case $m=3$ only.

Since about fifteen years, there is an increasing interest in the mathematical literature in higher-order nonlinear parabolic equations, due to their interesting mathematical structure and surprising properties. Probably the most famous example is the fourth-order thin-film equation

$$
u_{t}+\left(u^{\beta} u_{x x x}\right)_{x}=0, \quad \beta>0,
$$

which describes the surface-tension-dominated motion of thin viscous films for the film height $u(x, t)$ and spreading droplets in the lubrication approximation [14] or, for $\beta=1$, the thin neck of fluid in a Hele-Shaw cell [8]. This equation was first analyzed by Bernis and Friedman in [2]. An overview of some results can be found in [9]. Another interesting fourth-order equation arises in the context of spin systems [11]. Derrida et al. derived from a Toom model an equation which describes fluctuations of the interface between the regions of predominantly positive and negative particle spins, relative to the diagonal line:

$$
n_{t}+\left(n(\log n)_{x x}\right)_{x x}=0,
$$

where $n(x, t)$ is a suitable scaling limit of a random variable. Surprisingly, the same equation arises from the $O\left(\delta^{4}\right)$ approximation of the quantum drift-diffusion model mentioned above. The so-called Derrida-Lebowitz-Speer-Spohn equation (1.3) was first analzyed in [3] for local positive smooth solutions and then in [18] for global nonnegative weak solutions. We refer to [17] for a review on known results for (1.3).

Sixth-order nonlinear parabolic equations appear in flow modeling, describing the spreading of thin viscous droplets under different driving forces:

$$
u_{t}-\left(u^{\beta} u^{(\mathrm{v})}\right)_{x}=0, \quad \beta>0 .
$$

The case $\beta=3$, for instance, arises in a mathematical model of the oxidation of silicon in semiconductor devices [19] or for a moving boundary given by a beam of negligible mass on a surface of a thin film [24]. For a summary of mathematical results and more references we refer to [13]. Another sixth-order equation, with a higher-order $p$-Laplacian operator,

$$
u_{t}-\left(u^{\beta}\left|u^{(\mathrm{v})}\right|^{\gamma} u^{(\mathrm{v})}\right)_{x}=0, \quad \beta, \gamma>0,
$$

is obtained for power-law fluids spreading on a horizontal substrate [20]. Notice that these sixth-order equations are of degenerate type, whereas (1.1) is uniformly 
parabolic. Other (linear and nonlinear) sixth-order parabolic equations appear in cellular detonation formation dynamics [22], higher-order phase field models [5], and surface diffusion processes in the presence of strong anisotropy and curvature-dependent energy [4].

Most of the above mentioned higher-order models are physically positivity preserving, i.e., the solutions are expected to be nonnegative if they do so initially. For such equations, there is generally no maximum principle which makes the mathematical analysis and in particular the proof of nonnegativity quite difficult. General mathematical theories for these equations do not exist, and several principle questions concerning global existence, uniqueness, and regularity of solutions, in full generality, remain open. The mathematical difficulties for (1.1) come from the logarithmic terms, which complicates the proof of local existence of nonnegative solutions, and from the strong nonlinearities, which make it difficult to derive a priori estimates for global existence results. To our knowledge, no rigorous results are known up to now for the sixth-order equation (1.1).

In order to overcome the above mentioned problems, we employ two techniques. The first technique is to write (1.1) in different variables, such as the exponential variable $n=\exp y$ and the power variable $n=u^{4}$, and to solve (an approximation of) the resulting equation using fixed-point arguments. The advantage of both formulations is that for given (regular) $y$ or $u$, the original function $n$ becomes positive or nonnegative, respectively. More precisely, (1.1) is (for smooth positive solutions) equivalent to

$$
n_{t}-\left(n(\log n)_{x x x}\right)_{x x x}-2\left(n(\log n)_{x x}^{2}\right)_{x x}=0 .
$$

This formulation provides a symmetric structure in the sixth-order differential operator for the variable $y$, which is needed to solve the linearization in the fixed-point argument. Notice, however, that the fourth-order term may have a bad sign since the main part equals $-2\left(n_{x x} / n\right) n^{(\mathrm{iv})}$. This exponential variable transformation was also employed in $[16,18]$ for the fourth-order equation $(1.3)$. It is used here for the first time for a sixth-order equation.

We will prove the existence of solutions to an approximation of (1.4), but we are not able to prove the existence of solutions in the formulation (1.4) since there are seemingly no a priori bounds for $y=\log n$. We need to work with another formulation which avoids quotients and logarithms, and thus, both formulations (1.1) and (1.4) are not appropriate. It turns out that the right variable is $u=\sqrt[4]{n}$, since (1.1) can be written (again for smooth positive solutions) as

$$
\begin{aligned}
n_{t} & -2\left(\sqrt{n}(\sqrt{n})_{x x x}-\frac{16}{3} \sqrt[4]{n}(\sqrt[4]{n})_{x}^{3}\right)_{x x x}+12\left(\sqrt{n}(\sqrt[4]{n})_{x}^{2}\right)^{(\mathrm{iv})} \\
& -8\left((\sqrt{n})_{x x}^{2}+\frac{16}{3}(\sqrt[4]{n})_{x}^{4}\right)_{x x}=0 .
\end{aligned}
$$

We have formulated the second- and third-order derivatives in terms of $\sqrt{n}$ since the a priori estimate presented below provides a uniform $H^{3}$ bound for $\sqrt{n}(\cdot, t)$ and not for $\sqrt[4]{n}(\cdot, t)$. For $\sqrt[4]{n}(\cdot, t)$, only bounds for its first spatial derivative are available.

Our second technique is concerned with the derivation of a priori estimates needed in the existence analysis. From the physical application, we expect that the entropy functional

$$
E(n)=\int_{\mathbb{T}}(n(\log n-1)+1) d x
$$


is nonincreasing along the solutions to (1.1). The entropy dissipation $-d E / d t$, which is nonnegative, contains derivatives of the solution, thus providing gradient estimates. Such estimations can be obtained in principle by using $\log n$ as a test function in the weak formulation of (1.1) and by integrating by parts in a suitable way. For instance, we obtain from the formulation (1.4) for such a test function formally

$$
\frac{d E}{d t}+\int_{\mathbb{T}}\left(n(\log n)_{x x x}^{2}-2 n(\log n)_{x x}^{3}\right) d x=0
$$

but it is not clear how to estimate the third-order term $\left((\log n)_{x x}\right)^{3}$. The idea is to use systematic integration by parts, developed in [15] as the so-called algorithmic entropy construction method. In fact, it is shown in [15] that smooth positive solutions to (1.1) are satisfying the following estimate:

$$
\frac{d E}{d t}+C \int_{\mathbb{T}}\left((\sqrt{n})_{x x x}^{2}+(\sqrt[6]{n})_{x}^{6}\right) d x \leq 0
$$

where $C>0$ is some constant independent of $n$. In this paper, we make this estimate rigorous for weak nonnegative solutions and give an explicit value for the constant $C$. This so-called entropy-entropy dissipation inequality also allows us to prove the exponential decay of the solutions towards the steady state with an explicit rate involving $C$, valid for all $t>0$.

The entropy estimate (1.7) provides an $H^{3}$ bound for $\sqrt{n}(\cdot, t)$ and a $W^{1,6}$ estimate for $\sqrt[6]{n}(\cdot, t)$ (and $\sqrt[4]{n}(\cdot, t)$ ). Hence, we can interpret the formulation (1.5) in the sense of $H^{-3}(\mathbb{T})$. The existence proof for (1.5) is based on the solution of a certain approximated problem with strictly positive solutions $u_{\varepsilon}=\sqrt[4]{n_{\varepsilon}}$ indexed by a parameter $\varepsilon>0$. The difficulty is to perform the limit $\varepsilon \rightarrow 0$ in the approximated version of (1.5) and, in particular, to identify the weak limits. This is done by applying convergence results for transport plans from [1] (see Proposition 6.1 in the appendix).

Our main results are as follows.

THEOREM 1.1 (Existence of solutions). Let $T>0$ and let $n_{0}$ be a nonnegative measurable function on $\mathbb{T}$ with finite entropy $E\left(n_{0}\right)<\infty$. Then there exists a nonnegative weak solution $n$ to (1.2) and (1.5) satisfying

$$
\begin{aligned}
& n \in W^{1,5 / 4}\left(0, T ; H^{-3}(\mathbb{T})\right), \quad \sqrt{n} \in L^{2}\left(0, T ; H^{3}(\mathbb{T})\right), \\
& \sqrt[6]{n} \in L^{6}\left(0, T ; W^{1,6}(\mathbb{T})\right), \quad(\sqrt[4]{n})_{x}^{2} \in L^{2}\left(0, T ; H^{1}(\mathbb{T})\right) .
\end{aligned}
$$

Equation (1.5) and the initial condition (1.2) are satisfied in the sense of $L^{1}(0, T$; $\left.H^{-3}(\mathbb{T})\right)$.

THEOREM 1.2 (Exponential time decay). Let $n_{0}$ be a nonnegative measurable function on $\mathbb{T}$ with finite entropy $E\left(n_{0}\right)<\infty$ and unit mass $\int_{\mathbb{T}} n_{0} d x=1$. Let $n$ be the weak solution to (1.5) constructed in Theorem 1.1. Then, for all $t>0$,

$$
E(n(\cdot, t)) \leq \exp \left(-\frac{1}{2}(2 \pi)^{6} \kappa_{2} t\right) E\left(n_{0}\right)
$$

where $\kappa_{2}=2.73573 \ldots$ is the unique real solution to the third-order equation

$$
18 \kappa^{3}-199 \kappa^{2}+784 \kappa-1024=0 .
$$

Clearly, if the solution to (1.5) is positive and smooth, it solves the original formulation (1.1). 
By the Csiszár-Kullback inequality (see, e.g., [6]), we obtain the following decay in the $L^{1}$ norm:

$$
\|n(\cdot, t)-1\|_{L^{1}(\mathbb{T})} \leq \sqrt{2 E(n(\cdot, t))} \leq \exp \left(-\frac{1}{4}(2 \pi)^{6} \kappa_{2} t\right) \sqrt{2 E\left(n_{0}\right)} .
$$

It is shown in [12] that the entropy along the solutions to the fourth-order equation (1.3) decay exponentially fast to the mean value with rate $2(2 \pi)^{4}$. This rate seems to be optimal in view of the numerical results of [7]. The decay rate of the entropy of the sixth-order equation is larger than the rate for the fourth-order equation which is a consequence of the stronger diffusion.

The paper is organized as follows. In the following section, we prove two bounds from below for the integral in (1.6) providing some a priori estimates. The existence result is proved in Section 3 and the long-time behavior of the solutions is shown in Section 4. In section 5, we present some numerical examples, and the appendix is devoted to the proof of an auxiliary proposition, which may be of interest by itself, used to identify the weak limits.

2. Some auxiliary inequalities. In this section we show that the integral in (1.6) can be bounded from below by some gradient terms.

Lemma 2.1. Let $n \in H^{3}(\mathbb{T})$ satisfy $n>0$ in $\overline{\mathbb{T}}$. Then

$$
\int_{\mathbb{T}}\left(n(\log n)_{x x x}^{2}-2 n(\log n)_{x x}^{3}\right) d x \geq \kappa_{1} \int_{\mathbb{T}}\left((\sqrt{n})_{x x x}^{2}+(\sqrt[4]{n})_{x}^{2}(\sqrt[4]{n})_{x x}^{2}+(\sqrt[6]{n})_{x}^{6}\right) d x
$$

where $\kappa_{1}=2.72070 \ldots$ equals the smallest root of the fourth-order polynomial

$$
\frac{16379}{16384} \kappa^{4}-\frac{762195}{2048} \kappa^{3}+\frac{4153083}{1024} \kappa^{2}-\frac{254741}{16} \kappa+20736=0 .
$$

Recall that we employ the notation $u_{x}^{p}=\left(u_{x}\right)^{p}, u_{x x}^{p}=\left(u_{x x}\right)^{p}$ etc. for functions $u$ and numbers $p$. Notice that the inequality of the above lemma can be deduced from the results of [15] for some (non-explicit) constant $\kappa_{1}>0$. Lemma 2.1 provides such a constant, which may be useful to compute the explicit rate of decay to equilibrium of the solutions.

Proof. The proof is based on the algorithmic technique of [15]. The main idea of this method is to reformulate integration by parts as a decision problem for polynomials. We wish to find $\kappa>0$ such that the integral

$$
J=\int_{\mathbb{T}}\left(n(\log n)_{x x x}^{2}-2 n(\log n)_{x x}^{3}-\kappa(\sqrt{n})_{x x x}^{2}-\kappa(\sqrt[4]{n})_{x}^{2}(\sqrt[4]{n})_{x x}^{2}-\kappa(\sqrt[6]{n})_{x}^{6}\right) d x
$$

is nonnegative. To this end, we set $n=u^{4}$ and expand the derivatives:

$$
\begin{aligned}
J= & 16 \int_{\mathbb{T}} u^{4}\left[\left(12-\frac{4 \kappa}{729}\right)\left(\frac{u_{x}}{u}\right)^{6}-36\left(\frac{u_{x}}{u}\right)^{4} \frac{u_{x x}}{u}+4\left(\frac{u_{x}}{u}\right)^{3} \frac{u_{x x x}}{u}\right. \\
& +\left(33-\frac{37 \kappa}{16}\right)\left(\frac{u_{x}}{u}\right)^{2}\left(\frac{u_{x x}}{u}\right)^{2}-\left(6+\frac{3 \kappa}{2}\right) \frac{u_{x}}{u} \frac{u_{x x}}{u} \frac{u_{x x x}}{u}-8\left(\frac{u_{x x}}{u}\right)^{3} \\
& \left.+\left(1-\frac{\kappa}{4}\right)\left(\frac{u_{x x x}}{u}\right)^{2}\right] d x .
\end{aligned}
$$


In order to show the nonnegativity of the right-hand side, we employ the following integration-by-parts formulas:

$$
\begin{aligned}
& I_{1}=\int_{\mathbb{T}}\left(u^{4}\left(\frac{u_{x}}{u}\right)^{5}\right)_{x} d x=\int_{\mathbb{T}} u^{4}\left(-\left(\frac{u_{x}}{u}\right)^{6}+5\left(\frac{u_{x}}{u}\right)^{4} \frac{u_{x x}}{u}\right) d x \\
& I_{2}=\int_{\mathbb{T}}\left(u^{4}\left(\frac{u_{x}}{u}\right)^{3} \frac{u_{x x}}{u}\right)_{x} d x=\int_{\mathbb{T}} u^{4}\left(3\left(\frac{u_{x}}{u}\right)^{2}\left(\frac{u_{x x}}{u}\right)^{2}+\left(\frac{u_{x}}{u}\right)^{3} \frac{u_{x x}}{u}\right) d x, \\
& I_{3}=\int_{\mathbb{T}}\left(u^{4} \frac{u_{x}}{u}\left(\frac{u_{x x}}{u}\right)^{2}\right)_{x} d x=\int_{\mathbb{T}} u^{4}\left(\left(\frac{u_{x}}{u}\right)^{2}\left(\frac{u_{x x}}{u}\right)^{2}+\left(\frac{u_{x x}}{u}\right)^{3}+2 \frac{u_{x}}{u} \frac{u_{x x}}{u} \frac{u_{x x x}}{u}\right) d x .
\end{aligned}
$$

Clearly, in view of the periodic boundary conditions, $I_{1}=I_{2}=I_{3}=0$. There are more integration-by-parts formulas, but they involve derivatives of fourth and fifth order and are therefore not useful for the following analysis.

The idea of systematic integration by parts is to formulate the integrands of the above integrals as polynomials by translating the derivatives to polynomial variables:

$$
\xi_{1}=\frac{u_{x}}{u}, \quad \xi_{2}=\frac{u_{x x}}{u}, \quad \xi_{3}=\frac{u_{x x x}}{u} .
$$

Then the integrand of $J$, up to the factor $u^{4}$, translates to

$$
\begin{aligned}
S_{0}(\xi)= & \left(12-\frac{4 \kappa}{729}\right) \xi_{1}^{6}-36 \xi_{1}^{4} \xi_{2}+4 \xi_{1}^{3} \xi_{3}+\left(33-\frac{37 \kappa}{16}\right) \xi_{1}^{2} \xi_{2}^{2}-\left(6+\frac{3 \kappa}{2}\right) \xi_{1} \xi_{2} \xi_{3} \\
& -8 \xi_{2}^{3}+\left(1-\frac{\kappa}{4}\right) \xi_{3}^{2}
\end{aligned}
$$

where $\xi=\left(\xi_{1}, \xi_{2}, \xi_{3}\right)$, and the integrands of $I_{j}$ become

$$
T_{1}(\xi)=-\xi_{1}^{6}+5 \xi_{1}^{4} \xi_{2}, \quad T_{2}(\xi)=3 \xi_{1}^{2} \xi_{2}^{2}+\xi_{1}^{3} \xi_{3}, \quad T_{3}(\xi)=\xi_{1}^{2} \xi_{2}^{2}+\xi_{2}^{3}+2 \xi_{1} \xi_{2} \xi_{3} .
$$

These functions are called shift polynomials. Hence, the integral $J$ is nonnegative if there exist constants $c_{1}, c_{2}, c_{3} \in \mathbb{R}$ such that for all $\xi \in \mathbb{R}^{3}$,

$$
S(\xi)=\left(S_{0}+c_{1} T_{1}+c_{2} T_{2}+c_{3} T_{3}\right)(\xi) \geq 0 .
$$

The polynomial $S$ is indefinite in $\xi_{2}$ since the largest exponent is three. Therefore, we choose $c_{3}=8$ such that the coefficient of $\xi_{2}^{3}$ in $S$ vanishes. Then

$$
S(\xi)=a_{1} \xi_{1}^{6}+a_{2} \xi_{1}^{4} \xi_{2}+a_{3} \xi_{1}^{3} \xi_{3}+a_{4} \xi_{1}^{2} \xi_{2}^{2}+a_{5} \xi_{1} \xi_{2} \xi_{3}+a_{6} \xi_{3}^{2},
$$

where

$$
\begin{aligned}
& a_{1}=12-\frac{4 \kappa}{729}-c_{1}, \quad a_{2}=-36+5 c_{1}, \quad a_{3}=4+c_{2}, \\
& a_{4}=41-\frac{37 \kappa}{16}+3 c_{2}, \quad a_{5}=10-\frac{3 \kappa}{2}, \quad a_{6}=1-\frac{\kappa}{4} .
\end{aligned}
$$

The sign of the polynomial $S$ can be determined under the following conditions.

LEMMA 2.2 (Lemma 12 of [15]). Let the real polynomial

$$
S(\xi)=a_{1} \xi_{1}^{6}+a_{2} \xi_{1}^{4} \xi_{2}+a_{3} \xi_{1}^{3} \xi_{3}+a_{4} \xi_{1}^{2} \xi_{2}^{2}+a_{5} \xi_{1} \xi_{2} \xi_{3}+a_{6} \xi_{3}^{2}
$$

for $\xi=\left(\xi_{1}, \xi_{2}, \xi_{3}\right)$ be given and let $a_{6}>0$. Then the statement $S(\xi) \geq 0$ for all $\xi \in \mathbb{R}^{3}$ is equivalent to

$$
\begin{aligned}
& \text { either (i) } 4 a_{4} a_{6}-a_{5}^{2}=2 a_{2} a_{6}-a_{3} a_{5}=0 \text { and } 4 a_{1} a_{6}-a_{3}^{2} \geq 0, \\
& \quad \text { or (ii) } 4 a_{4} a_{6}-a_{5}^{2}>0 \text { and } 4 a_{1} a_{4} a_{6}-a_{1} a_{5}^{2}-a_{2}^{2} a_{6}-a_{3}^{2} a_{4}+a_{2} a_{3} a_{5} \geq 0 .
\end{aligned}
$$


We remark that the condition $a_{6}>0$ is equivalent to $\kappa<4$. The equations $4 a_{4} a_{6}-a_{5}^{2}=0$ and $2 a_{2} a_{6}-a_{3} a_{5}=0$ are a linear system in the variables $c_{1}$ and $c_{2}$,

$$
\begin{array}{r}
3(4-\kappa) c_{2}+\frac{\kappa^{2}}{16}-\frac{81}{4} \kappa+64=0, \\
\left(10-\frac{5}{2} \kappa\right) c_{1}-\left(10-\frac{3}{2} \kappa\right) c_{2}+24 \kappa-112=0 .
\end{array}
$$

Inserting the unique solution $\left(c_{1}, c_{2}\right)$ of this system into the inequality of (i) yields the following condition on $\kappa$ :

$$
\frac{16379}{16384} \kappa^{4}-\frac{762195}{2048} \kappa^{3}+\frac{4153083}{1024} \kappa^{2}-\frac{254741}{16} \kappa+20736 \geq 0 .
$$

This inequality is satisfied if and only if $\kappa$ is smaller than the smaller root $\kappa_{1}^{*}=$ $2.72070 \ldots$ or larger than the larger root $\kappa_{2}^{*}=361.16783 \ldots$ In view of the condition $0<\kappa<4$, we conclude that all $\kappa \leq \kappa_{1}^{*}$ are admissible. We notice that condition (ii) does not provide a better result.

The constant $\kappa_{1}$ in Lemma 2.1 can be slightly improved if we take into account the dissipation $\int_{\mathbb{T}}(\sqrt{n})_{x x x}^{2} d x$ only. This result will be used for the computation of the decay rate of Theorem 1.2.

Lemma 2.3. Let $n \in H^{3}(\mathbb{T})$ satisfy $n>0$ in $\overline{\mathbb{T}}$. Then

$$
\int_{\mathbb{T}}\left(n(\log n)_{x x x}^{2}-2 n(\log n)_{x x}^{3}\right) d x \geq \kappa_{2} \int_{\mathbb{T}}(\sqrt{n})_{x x x}^{2} d x,
$$

where $\kappa_{2}$ is defined in (1.8).

Proof. We wish to find $\kappa>0$ such that

$$
\begin{aligned}
J= & \int_{\mathbb{T}}\left(n(\log n)_{x x x}^{2}-2 n(\log n)_{x x}^{3}-\kappa(\sqrt{n})_{x x x}^{2}\right) d x \\
= & 16 \int_{\mathbb{T}} u^{4}\left[12\left(\frac{u_{x}}{u}\right)^{6}-36\left(\frac{u_{x}}{u}\right)^{4} \frac{u_{x x}}{u}+4\left(\frac{u_{x}}{u}\right)^{3} \frac{u_{x x x}}{u}\right. \\
& +\left(33-\frac{9}{4} \kappa\right)\left(\frac{u_{x}}{u}\right)^{2}\left(\frac{u_{x x}}{u}\right)^{2}-\left(6+\frac{3}{2} \kappa\right) \frac{u_{x}}{u} \frac{u_{x x}}{u} \frac{u_{x x x}}{u}-8\left(\frac{u_{x x}}{u}\right)^{3} \\
& \left.+\left(1-\frac{\kappa}{4}\right)\left(\frac{u_{x x x}}{u}\right)^{2}\right] d x \geq 0 .
\end{aligned}
$$

With the notations of the previous proof, we associate to the integrand (up to the factor $u^{4}$ ) the polynomial

$$
\begin{aligned}
S_{0}(\xi)= & 12 \xi_{1}^{6}-36 \xi_{1}^{4} \xi_{2}+4 \xi_{1}^{3} \xi_{3}+\left(33-\frac{9}{4} \kappa\right) \xi_{1}^{2} \xi_{2}^{2}-\left(6+\frac{3}{2} \kappa\right) \xi_{1} \xi_{2} \xi_{3}-8 \xi_{2}^{3} \\
& +\left(1-\frac{\kappa}{4}\right) \xi_{3}^{2} .
\end{aligned}
$$

Employing the same shift polynomials $T_{i}$ as in the previous proof, the integral $J$ is nonnegative if there are constants $c_{1}, c_{2}, c_{3} \in \mathbb{R}$ such that for all $\xi \in \mathbb{R}^{3}$,

$$
S(\xi)=\left(S_{0}+c_{1} T_{1}+c_{2} T_{2}+c_{3} T_{3}\right)(\xi) \geq 0 .
$$

We choose as in the previous proof $c_{3}=8$ in order to eliminate the term $\xi_{2}^{3}$. This gives the polynomial

$$
S(\xi)=a_{1} \xi_{1}^{6}+a_{2} \xi_{1}^{4} \xi_{2}+a_{3} \xi_{1}^{3} \xi_{3}+a_{4} \xi_{1}^{2} \xi_{2}^{2}+a_{5} \xi_{1} \xi_{2} \xi_{3}+a_{6} \xi_{3}^{2},
$$


where

$$
\begin{aligned}
& a_{1}=12-c_{1}, \quad a_{2}=-36+5 c_{1}, \quad a_{3}=4+c_{2}, \\
& a_{4}=41-\frac{9}{4} \kappa+3 c_{2}, \quad a_{5}=10-\frac{3}{2} \kappa, \quad a_{6}=1-\frac{\kappa}{4} .
\end{aligned}
$$

The two equations of condition (i) in Lemma 2.2 are linear equations for $c_{1}$ and $c_{2}$,

$$
\begin{aligned}
3(4-\kappa) c_{2}-20 \kappa+64 & =0 \\
\left(10-\frac{5}{2} \kappa\right) c_{1}+\left(\frac{3}{2} \kappa-10\right) c_{2}+24 \kappa-112 & =0 .
\end{aligned}
$$

Inserting the unique solution $\left(c_{1}, c_{2}\right)$ into the inequality in condition (i) yields the following inequality for $\kappa$ :

$$
18 \kappa^{3}-199 \kappa^{2}+784 \kappa-1024 \leq 0 .
$$

All $\kappa \leq \kappa_{2}$, where $\kappa_{2}$ is the unique solution to (1.8), satisfy this inequality. We notice that condition (ii) gives the stronger restriction $\kappa<\kappa_{2}$.

3. Existence of solutions. This section is devoted to the proof of Theorem 1.1. The proof is divided in several steps.

3.1. Existence of a time-discrete problem. Let $T>0$ and $\tau>0$ be given. We wish to find, for given $w$, a solution $u \in W^{1,6}(\mathbb{T})$ with $u^{2} \in H^{3}(\mathbb{T})$ to the semidiscrete equation

$$
\begin{aligned}
\frac{1}{\tau}\left(u^{4}-w^{4}\right)= & 2\left(u^{2}\left(u^{2}\right)_{x x x}-\frac{16}{3} u u_{x}^{3}\right)_{x x x}-12\left(u^{2} u_{x}^{2}\right)^{(\mathrm{iv})} \\
& +8\left(\left(u^{2}\right)_{x x}^{2}+\frac{16}{3} u_{x}^{4}\right)_{x x}
\end{aligned}
$$

LEMMA 3.1. Let $w$ be a nonnegative measurable function satisfying $E\left(w^{4}\right)<\infty$ and $\left\|w^{4}\right\|_{L^{1}(\mathbb{T})}=1$. Then there exists a weak solution $u \in W^{1,6}(\mathbb{T})$ to $(3.1)$ satisfying $u^{2} \in H^{3}(\mathbb{T})$ and $u_{x}^{2} \in H^{1}(\mathbb{T})$. Furthermore, $u^{4}$ has unit mass and the physical entropy is dissipated in the sense

$$
E\left(u^{4}\right)+\tau C \int_{\mathbb{T}}\left(\left(u^{2}\right)_{x x x}^{2}+\left(u_{x}^{2}\right)_{x}^{2}+u_{x}^{6}\right) d x \leq E\left(w^{4}\right),
$$

where $C>0$ is a constant independent of $\tau$.

Proof. In the proof we work simultaneously in the variables $u$ and $y=\log \left(u^{4}\right)$.

Step 1: solution of a regularized problem. We define first a regularized problem. The solution to (3.1) is then obtained as the limit of the solutions to the regularized problem. We reformulate $(3.1)$ by introducing the logarithmic variable $y=\log \left(u^{4}\right)$ as

$$
\frac{1}{\tau}\left(u^{4}-w^{4}\right)=\left(u^{4} y_{x x x}\right)_{x x x}+32\left(u^{2} u_{x x}^{2}-2 u u_{x}^{2} u_{x x}+u_{x}^{4}\right)_{x x}+\varepsilon\left(y^{(v i)}-y\right),
$$

where $\varepsilon>0$ is a regularization parameter. The first term on the right-hand side comes from the formulation (1.4). The second term equals

$$
32\left(u^{2} u_{x x}^{2}-2 u u_{x}^{2} u_{x x}+u_{x}^{4}\right)_{x x}=2\left(u^{4}\left(\log \left(u^{4}\right)\right)_{x x}^{2}\right)_{x x} .
$$

The third term guarantees coercivity of the right-hand side with respect to $y$. 
In order to solve (3.3), we employ the Leray-Schauder fixed-point theorem (see, e.g., $\left[25\right.$, Theorem B.5]). Let $\sigma \in[0,1]$ and $v \in W^{2,4}(\mathbb{T})$. We define for $y, z \in H^{3}(\mathbb{T})$,

$$
\begin{aligned}
a(y, z) & =\int_{\mathbb{T}}\left(\sigma v^{4} y_{x x x} z_{x x x}+\varepsilon y_{x x x} z_{x x x}+\varepsilon y z\right) d x, \\
f(z) & =-\frac{\sigma}{\tau} \int_{\mathbb{T}}\left(v^{4}-w^{4}\right) z d x+32 \sigma \int_{\mathbb{T}}\left(v^{2} v_{x x}^{2}-2 v v_{x}^{2} v_{x x}+v_{x}^{4}\right) z_{x x} d x .
\end{aligned}
$$

The bilinear form $a$ is continuous and coercive since

$$
a(y, y) \geq \varepsilon \int_{\mathbb{T}}\left(y_{x x x}^{2}+y^{2}\right) d x \geq C \varepsilon\|y\|_{H^{3}(\mathbb{T})}^{2},
$$

where $C>0$ is some constant. Moreover, $f$ is continuous on $H^{3}(\mathbb{T})$ since $w^{4} \in$ $L^{1}(\mathbb{T}) \hookrightarrow H^{-1}(\mathbb{T})$ and $v \in W^{2,4}(\mathbb{T}) \hookrightarrow L^{\infty}(\mathbb{T})$ implies that $v^{2} v_{x x}^{2}-2 v v_{x}^{2} v_{x x}+v_{x}^{4} \in$ $L^{2}(\mathbb{T})$. Therefore, the Lax-Milgram lemma provides the existence of a unique solution $y \in H^{3}(\mathbb{T})$ to the linear problem

$$
a(y, z)=f(z) \quad \text { for all } z \in H^{3}(\mathbb{T}) .
$$

This defines the fixed-point operator $S: W^{2,4}(\mathbb{T}) \times[0,1] \rightarrow W^{2,4}(\mathbb{T}), S(v, \sigma)=u=$ $e^{y / 4}$. Notice that in one space dimension, $H^{3}(\mathbb{T})$ embeddes continuously into $W^{2,4}(\mathbb{T})$ and thus, $S$ is well-defined. It holds $S(v, 0)=1$. By standard results for elliptic equations, $S$ is continuous and compact since the embedding $H^{3}(\mathbb{T}) \hookrightarrow W^{2,4}(\mathbb{T})$ is compact. It remains to prove a uniform bound for all fixed points of $S(\cdot, \sigma)$.

Let $u \in H^{3}(\mathbb{T})$ be a fixed point of $S(\cdot, \sigma)$ for some $\sigma \in[0,1]$. Then, by construction, $u=e^{y / 4}>0$ is a solution to (3.3). The convexity of $\phi(s)=s(\log s-1)+1$ implies that

$$
\begin{aligned}
\frac{1}{\tau} & \left(E\left(u^{4}\right)-E\left(w^{4}\right)\right)=\frac{1}{\tau} \int_{\mathbb{T}}\left(\phi\left(u^{4}\right)-\phi\left(w^{4}\right)\right) d x \\
& \leq \frac{1}{\tau} \int_{\mathbb{T}} \phi^{\prime}\left(u^{4}\right)\left(u^{4}-w^{4}\right) d x=\frac{1}{\tau} \int_{\mathbb{T}} \log \left(u^{4}\right)\left(u^{4}-w^{4}\right) d x \\
& =\int_{\mathbb{T}}\left(-u^{4} y_{x x x}^{2}+32\left(u^{2} u_{x x}^{2}-2 u u_{x}^{2} u_{x x}+u_{x}^{4}\right) y_{x x}\right) d x-\varepsilon \int_{\mathbb{T}}\left(y_{x x x}^{2}+y^{2}\right) d x \\
& =-\int_{\mathbb{T}}\left(u^{4}\left(\log \left(u^{4}\right)\right)_{x x x}^{2}-2 u^{4}\left(\log \left(u^{4}\right)\right)_{x x}^{3}\right) d x-\varepsilon \int_{\mathbb{T}}\left(y_{x x x}^{2}+y^{2}\right) d x .
\end{aligned}
$$

Lemma 2.1 shows that

$$
\int_{\mathbb{T}}\left(u^{4}\left(\log \left(u^{4}\right)\right)_{x x x}^{2}-2 u^{4}\left(\log \left(u^{4}\right)\right)_{x x}^{3}\right) d x \geq \kappa_{1} \int_{\mathbb{T}}\left(\left(u^{2}\right)_{x x x}^{2}+u_{x}^{2} u_{x x}^{2}+\left(u^{2 / 3}\right)_{x}^{6}\right) d x,
$$

giving the estimate

$$
\begin{gathered}
E\left(u^{4}\right)+\tau \kappa_{1} \int_{\mathbb{T}}\left(\left(u^{2}\right)_{x x x}^{2}+u_{x}^{2} u_{x x}^{2}+\left(u^{2 / 3}\right)_{x}^{6}\right) d x \\
+\tau \varepsilon \int_{\mathbb{T}}\left(y_{x x x}^{2}+y^{2}\right) d x \leq E\left(w^{4}\right) .
\end{gathered}
$$

Thus, $y$ and $y_{x x x}$ are bounded in $L^{2}(\mathbb{T})$ (with a bound which depends on $\varepsilon$ ). By the Gagliardo-Nirenberg inequality, we infer a bound for $y$ in $H^{3}(\mathbb{T})$. This shows that 
$u=e^{y / 4}$ is bounded in $H^{3}(\mathbb{T})$ and hence, also in $W^{2,4}(\mathbb{T})$. This proves the desired bound for all fixed points of $S(\cdot, \sigma)$.

The Leray-Schauder theorem then provides a solution $u$ to $S(u, 1)=u$, which we denote by $u_{\varepsilon}$. By construction, we can write $u_{\varepsilon}=\exp \left(y_{\varepsilon} / 4\right)>0$ for some $y_{\varepsilon} \in H^{3}(\mathbb{T})$. This fixed point is a periodic solution to the following equation:

$$
\begin{aligned}
\frac{1}{\tau}\left(u_{\varepsilon}^{4}-w^{4}\right)= & \left(u_{\varepsilon}^{4}\left(\log u_{\varepsilon}^{4}\right)_{x x x}\right)_{x x x}+32\left(u_{\varepsilon}^{2} u_{\varepsilon, x x}^{2}-2 u_{\varepsilon} u_{\varepsilon, x}^{2} u_{\varepsilon, x x}+u_{\varepsilon, x}^{4}\right)_{x x} \\
& +\varepsilon\left(y_{\varepsilon}^{(\mathrm{vi})}-y_{\varepsilon}\right),
\end{aligned}
$$

which can be written equivalently as

$$
\begin{aligned}
\frac{1}{\tau}\left(u_{\varepsilon}^{4}-w^{4}\right)= & 2\left(u_{\varepsilon}^{2}\left(u_{\varepsilon}^{2}\right)_{x x x}-\frac{16}{3} u_{\varepsilon} u_{\varepsilon, x}^{3}\right)_{x x x}-12\left(u_{\varepsilon}^{2} u_{\varepsilon, x}^{2}\right)^{(\mathrm{iv})} \\
& +8\left(\left(u_{\varepsilon}^{2}\right)_{x x}^{2}+\frac{16}{3} u_{\varepsilon, x}^{4}\right)_{x x}+\varepsilon\left(y_{\varepsilon}^{(\mathrm{vi})}-y_{\varepsilon}\right) .
\end{aligned}
$$

Step 2: limit $\varepsilon \rightarrow 0$. We have to show that, as $\varepsilon \rightarrow 0, u_{\varepsilon}$ converges to some function $u$, which solves (3.1). The estimate (3.4) shows that the sequence $\left(u_{\varepsilon}^{2 / 3}\right)$ is bounded in $W^{1,6}(\mathbb{T})$ and that $\left(u_{\varepsilon}^{2}\right)$ is bounded in $H^{3}(\mathbb{T})$. Since $W^{1,6}(\mathbb{T})$ embeddes continuously into $L^{\infty}(\mathbb{T})$, the first bound implies that also $\left(u_{\varepsilon}\right)$ is bounded in $W^{1,6}(\mathbb{T})$. Thus, for a subsequence, as $\varepsilon \rightarrow 0$,

$$
u_{\varepsilon} \rightarrow u \quad \text { weakly in } W^{1,6}(\mathbb{T}), \quad u_{\varepsilon}^{2} \rightarrow g \quad \text { weakly in } H^{3}(\mathbb{T}) .
$$

Employing again the compactness embedding of $W^{1,6}(\mathbb{T})$ into $L^{\infty}(\mathbb{T})$, again for a subsequence, we infer that $u_{\varepsilon} \rightarrow u$ strongly in $L^{\infty}(\mathbb{T})$. Hence, $g=u^{2}$. Moreover, $u_{\varepsilon}^{2} \rightarrow u^{2}$ strongly in $W^{2, \infty}(\mathbb{T})$. These results show that

$$
\begin{aligned}
u_{\varepsilon}^{2}\left(u_{\varepsilon}^{2}\right)_{x x x} \rightarrow u^{2}\left(u^{2}\right)_{x x x} & \text { weakly in } L^{2}(\mathbb{T}), \\
\left(u_{\varepsilon}^{2}\right)_{x x}^{2} \rightarrow\left(u^{2}\right)_{x x}^{2} & \text { strongly in } L^{\infty}(\mathbb{T}),
\end{aligned}
$$

and

$$
\left(u_{\varepsilon}^{2} u_{\varepsilon, x}^{2}\right)_{x}=\frac{1}{2}\left(u_{\varepsilon}^{2}\right)_{x}\left(u_{\varepsilon}^{2}\right)_{x x} \rightarrow \frac{1}{2}\left(u^{2}\right)_{x}\left(u^{2}\right)_{x x}=\frac{1}{4}\left(\left(u^{2}\right)_{x}^{2}\right)_{x}=\left(u^{2} u_{x}^{2}\right)_{x}
$$

strongly in $L^{\infty}(\mathbb{T})$. By Proposition 6.1 of the appendix, with $\alpha=2, \beta=2 / 3$, and $\gamma=4 / 3$, and the $W^{1,6}$ bound for $u_{\varepsilon}$,

$$
u_{\varepsilon} u_{\varepsilon, x}^{3}=\left(\frac{3}{4}\right)^{3}\left(u_{\varepsilon}^{4 / 3}\right)_{x}^{3} \rightarrow\left(\frac{3}{4}\right)^{3}\left(u^{4 / 3}\right)_{x}^{3}=u u_{x}^{3} \quad \text { strongly in } L^{2}(\Omega),
$$

and, with $\alpha=2, \beta=2 / 3, \gamma=1$,

$$
u_{\varepsilon, x}^{4} \rightarrow u_{x}^{4} \quad \text { strongly in } L^{3 / 2}(\Omega) .
$$

Finally, the entropy estimate (3.4) shows that $\sqrt{\varepsilon}\left\|y_{\varepsilon}\right\|_{H^{3}(\mathbb{T})}$ is uniformly bounded. Hence, for a subsequence,

$$
\varepsilon y_{\varepsilon} \rightarrow 0 \quad \text { strongly in } H^{3}(\mathbb{T}) \text {. }
$$

The convergence results (3.7)-(3.12) allow us to perform the limit $\varepsilon \rightarrow 0$ in (3.5), which holds in the sense of $H^{-4}(\mathbb{T})$. Therefore, $u$ is a nonnegative weak solution to (3.1). 
Step 3: end of the proof. We need to verify that $u^{4}$ has unit mass and that (3.2) holds. Conservation of mass follows from the weak formulation of (3.1) by using $z=1$ as a test function. It follows from (3.4) that $u_{\varepsilon}$ satisfies, for some constant $C>0$,

$$
E\left(u_{\varepsilon}^{4}\right)+\tau C \int_{\mathbb{T}}\left(\left(u_{\varepsilon}^{2}\right)_{x x x}^{2}+u_{\varepsilon, x}^{6}+\left(u_{\varepsilon, x}^{2}\right)_{x}^{2}\right) d x \leq E\left(w^{4}\right) .
$$

Since $\left(u_{\varepsilon}^{2}\right)_{x x x}, u_{\varepsilon, x}^{3}$, and $\left(u_{\varepsilon, x}^{2}\right)_{x}$ converge weakly in $L^{2}(\mathbb{T})$, we conclude that

$$
\begin{aligned}
E\left(u^{4}\right) & +\tau C \int_{\mathbb{T}}\left(\left(u^{2}\right)_{x x x}^{2}+u_{x}^{6}+\left(u_{x}^{2}\right)_{x}^{2}\right) d x \\
& \leq E\left(u^{4}\right)+\tau C \liminf _{\varepsilon \rightarrow 0} \int_{\mathbb{T}}\left(\left(u_{\varepsilon}^{2}\right)_{x x x}^{2}+u_{\varepsilon, x}^{6}+\left(u_{\varepsilon, x}^{2}\right)_{x}^{2}\right) d x \leq E\left(w^{4}\right) .
\end{aligned}
$$

This finishes the proof.

REMARK 3.2 (Convergence). The convergence results (3.10) and (3.11) can be proved without the use of Proposition 6.1 by employing the $H^{1}$ bound for $u_{\varepsilon, x}^{2}$. Indeed, this fact, together with the boundedness of $\left(u_{\varepsilon, x}\right)$ in $L^{6}(\mathbb{T})$, implies that the sequence $\left(u_{\varepsilon, x}^{3}\right)_{x}=\frac{3}{2} u_{\varepsilon, x}\left(u_{\varepsilon, x}^{2}\right)_{x}$ is bounded in $L^{3 / 2}(\mathbb{T})$. In particular, $\left(u_{\varepsilon, x}^{3}\right)$ is bounded in $W^{1,3 / 2}(\mathbb{T})$, which embeddes compactly into $L^{\infty}(\mathbb{T})$. Thus, for a subsequence, $u_{\varepsilon, x}^{3} \rightarrow h$ and $u_{\varepsilon, x} \rightarrow \sqrt[3]{h}$ strongly in $L^{\infty}(\mathbb{T})$ for some function $h$. By (3.6), $u_{\varepsilon, x} \rightarrow u_{x}$ weakly in $L^{6}(\mathbb{T})$. We conclude that $\sqrt[3]{h}=u_{x}$ and hence, $u_{\varepsilon, x}^{3} \rightarrow u_{x}^{3}$ strongly in $L^{\infty}(\mathbb{T})$ and $u_{\varepsilon, x}^{3} \rightarrow u_{x}^{3}$ weakly in $W^{1,3 / 2}(\mathbb{T})$. Therefore,

$$
\begin{aligned}
u_{\varepsilon} u_{\varepsilon, x}^{3} \rightarrow u u_{x}^{3} & \text { strongly in } L^{\infty}(\mathbb{T}), \\
u_{\varepsilon, x}^{4}=u_{\varepsilon, x} u_{\varepsilon, x}^{3} \rightarrow u_{x} u_{x}^{3}=u_{x}^{4} & \text { weakly in } L^{6}(\mathbb{T}),
\end{aligned}
$$

which is sufficient to conclude the proof.

REMARK 3.3 (Regularity). Lemma 3.1 shows that the square root of the particle density satisfies the regularity $\sqrt{n}=u^{2} \in H^{3}(\mathbb{T})$. Actually, we can show a slightly improved result. We claim that $n=u^{4} \in H^{4}(\mathbb{T})$. In order to see this, we observe that (3.1) can be rewritten, in the sense of distributions, as

$$
\frac{1}{\tau}\left(u^{4}-w^{4}\right)=\left(u^{4}\right)^{(\mathrm{vi})}-4\left[3\left(u^{2}\right)_{x}\left(u^{2}\right)_{x x x}-4\left(u^{2}\right)_{x x} u_{x}^{2}+\left(u^{2}\right)_{x x}^{2}+4\left(u^{2}\right)_{x}\left(u_{x}^{2}\right)_{x}\right]_{x x} .
$$

Since the terms inside of the bracket $[\cdots]$ are $L^{2}(\mathbb{T})$ functions, we infer that $\left(u^{4}\right)^{(\mathrm{vi})}$ $\in H^{-2}(\mathbb{T})$ and therefore $u^{4} \in H^{4}(\mathbb{T})$.

It is known that whenever a function $u^{2}$ has bounded second-order derivatives, then $u$ has bounded first-order derivatives [21] and

$$
\left\|u_{x}\right\|_{L^{\infty}(\mathbb{T})} \leq c\left\|\left(u^{2}\right)_{x x}\right\|_{L^{\infty}(\mathbb{T})}
$$

for some constant $c>0$. Now, since $u^{2} \in H^{3}(\mathbb{T}) \hookrightarrow W^{2, \infty}(\mathbb{T})$, we obtain by the above result that $\sqrt[4]{n}=u \in W^{1, \infty}(\mathbb{T})$.

3.2. A priori estimates. Let $T>0$ and $\tau>0$ be given such that $T / \tau \in \mathbb{N}$. We define the step function $u^{(\tau)}:[0, T) \rightarrow L^{2}(\mathbb{T})$ recursively as follows. Let $u_{0}=\sqrt[4]{n_{0}}$ and for given $k \in \mathbb{N}$, let $u_{k} \in W^{1,6}(\mathbb{T})$ with $u^{2} \in H^{3}(\mathbb{T})$ be a nonnegative solution to (3.1) with $w=u_{k-1}$. Now define $u^{(\tau)}(t)=u_{k}$ for $(k-1) \tau<t \leq k \tau$. Then $u^{(\tau)}$ 
satisfies the equation

$$
\begin{aligned}
\frac{1}{\tau}\left(\left(u^{(\tau)}\right)^{4}-\right. & \left.\left(\sigma_{\tau} u^{(\tau)}\right)^{4}\right)=2\left(\left(u^{(\tau)}\right)^{2}\left(\left(u^{(\tau)}\right)^{2}\right)_{x x x}-\frac{16}{3} u^{(\tau)}\left(u^{(\tau)}\right)_{x}^{3}\right)_{x x x} \\
- & 12\left(\left(u^{(\tau)}\right)^{2}\left(u^{(\tau)}\right)_{x}^{2}\right)^{(\mathrm{iv})}+8\left(\left(\left(u^{(\tau)}\right)^{2}\right)_{x x}^{2}+\frac{16}{3}\left(u^{(\tau)}\right)_{x}^{4}\right)_{x x}
\end{aligned}
$$

where $\sigma_{\tau}$ denotes the shift operator $\left(\sigma_{\tau} u^{(\tau)}\right)(t)=u^{(\tau)}(t-\tau)$ for $\tau \leq t<T$. By (3.2) and taking into account the elementary inequality $x^{4}\left(\log x^{4}-1\right) \geq x-e$ for all $x \geq 0$, we obtain the following a priori estimates:

$$
\begin{array}{r}
\left\|u^{(\tau)}\right\|_{L^{\infty}\left(0, T ; L^{4}(\mathbb{T})\right)}+\left\|\left(u^{(\tau)}\right)^{2}\right\|_{L^{2}\left(0, T ; H^{3}(\mathbb{T})\right)} \leq C, \\
\left\|\left(u^{(\tau)}\right)_{x}^{2}\right\|_{L^{2}\left(0, T ; H^{1}(\mathbb{T})\right)}+\left\|u^{(\tau)}\right\|_{L^{6}\left(0, T ; W^{1,6}(\mathbb{T})\right)} \leq C,
\end{array}
$$

where $C>0$ denotes here and in the following a generic constant not depending on $\tau$. For the limit $\tau \rightarrow 0$, we need more estimates.

LEMMA 3.4. The function $u^{(\tau)}$ satisfies

$$
\tau^{-1}\left\|\left(u^{(\tau)}\right)^{4}-\left(\sigma_{\tau} u^{(\tau)}\right)^{4}\right\|_{L^{5 / 4}\left(0, T ; H^{-3}(\mathbb{T})\right)} \leq C .
$$

Proof. We have to show that the right-hand side of (3.13) is uniformly bounded in $L^{5 / 4}\left(0, T ; H^{-3}(\mathbb{T})\right)$. To simplify the notation, we write $u=u^{(\tau)}$. First, we remark that by the Gagliardo-Nirenberg inequality, it holds for $\theta=1 / 6$,

$$
\begin{aligned}
\|u\|_{L^{24}\left(0, T ; L^{\infty}(\mathbb{T})\right)}^{24} & \leq C \int_{0}^{T}\left\|u(\cdot, t)^{2}\right\|_{H^{3}(\mathbb{T})}^{12 \theta}\left\|u(\cdot, t)^{2}\right\|_{L^{2}(\mathbb{T})}^{12(1-\theta)} d t \\
& \leq C\|u\|_{L^{\infty}\left(0, T ; L^{4}(\mathbb{T})\right)}^{24(1-\theta)} \int_{0}^{T}\left\|u^{2}(\cdot, t)\right\|_{H^{3}(\mathbb{T})}^{12 \theta} d t \leq C .
\end{aligned}
$$

Now we estimate the right-hand side of (3.13) term by term. By the Hölder inequality with respect to $t$, applied for $p=8 / 3$ and $p^{\prime}=8 / 5$, we obtain

$$
\begin{aligned}
\left\|u^{2}\left(u^{2}\right)_{x x x}\right\|_{L^{5 / 4}\left(0, T ; L^{2}(\mathbb{T})\right)}^{5 / 4} & \leq \int_{0}^{T}\|u(\cdot, t)\|_{L^{\infty}(\mathbb{T})}^{5 / 2}\left\|u(\cdot, t)^{2}\right\|_{H^{3}(\mathbb{T})}^{5 / 4} d t \\
& \leq\|u\|_{L^{20 / 3}\left(0, T ; L^{\infty}(\mathbb{T})\right)}^{5 / 2}\left\|u^{2}\right\|_{L^{2}\left(0, T ; H^{3}(\mathbb{T})\right)}^{5 / 4} \leq C,
\end{aligned}
$$

taking into account the estimates (3.14) and (3.17). Applying the Hölder inequality with $p=8 / 3$ and $p^{\prime}=8 / 5$ yields

$$
\begin{aligned}
\left\|u u_{x}^{3}\right\|_{L^{5 / 4}\left(0, T ; L^{2}(\mathbb{T})\right)}^{5 / 4} & \leq \int_{0}^{T}\|u(\cdot, t)\|_{L^{\infty}(\mathbb{T})}^{5 / 4}\|u(\cdot, t)\|_{W^{1,6}(\mathbb{T})}^{15 / 4} d t \\
& \leq\|u\|_{L^{10 / 3}\left(0, T ; L^{\infty}(\mathbb{T})\right)}^{5 / 4}\|u\|_{L^{6}\left(0, T ; W^{1,6}(\mathbb{T})\right)}^{15 / 4} \leq C,
\end{aligned}
$$

in view of (3.15) and (3.17). The estimates (3.14), (3.15), and (3.17) show that

$$
\begin{aligned}
& \left\|\left(u^{2} u_{x}^{2}\right)_{x}\right\|_{L^{5 / 4}\left(0, T ; L^{2}(\mathbb{T})\right)}=\left\|u u_{x}\left(u^{2}\right)_{x x}\right\|_{L^{5 / 4}\left(0, T ; L^{2}(\mathbb{T})\right)} \\
& \quad \leq\|u\|_{L^{15 / 2}\left(0, T ; L^{\infty}(\mathbb{T})\right)}\left\|u_{x}\right\|_{L^{6}\left(0, T ; L^{6}(\mathbb{T})\right)}\left\|\left(u^{2}\right)_{x x}\right\|_{L^{2}\left(0, T ; L^{2}(\mathbb{T})\right)} \leq C .
\end{aligned}
$$


Next, we apply the Gagliardo-Nirenberg inequality with $\theta=7 / 10$ and then the Hölder inequality with $p=8 / 7$ and $p^{\prime}=8$ :

$$
\begin{aligned}
\left\|\left(u^{2}\right)_{x x}^{2}\right\|_{L^{5 / 4}\left(0, T ; L^{2}(\mathbb{T})\right)}^{5 / 4} & =\left\|\left(u^{2}\right)_{x x}\right\|_{L^{5 / 2}\left(0, T ; L^{4}(\mathbb{T})\right)}^{5 / 2} \\
& \leq C \int_{0}^{T}\left\|u(\cdot, t)^{2}\right\|_{H^{3}(\mathbb{T})}^{5 \theta / 2}\left\|u(\cdot, t)^{2}\right\|_{L^{\infty}(\mathbb{T})}^{5(1-\theta) / 2} d t \\
& =C \int_{0}^{T}\left\|u(\cdot, t)^{2}\right\|_{H^{3}(\mathbb{T})}^{7 / 4}\|u(\cdot, t)\|_{L^{\infty}(\mathbb{T})}^{3 / 2} d t \\
& \leq C\left\|u^{2}\right\|_{L^{2}\left(0, T ; H^{3}(\mathbb{T})\right)}^{7 / 4}\|u\|_{L^{12}\left(0, T ; L^{\infty}(\mathbb{T})\right)}^{3 / 2} \leq C,
\end{aligned}
$$

by (3.14) and (3.17). Finally, we conclude from (3.15) that

$$
\left\|u_{x}^{4}\right\|_{L^{5 / 4}\left(0, T ; L^{1}(\mathbb{T})\right)}=\left\|u_{x}\right\|_{L^{5}\left(0, T ; L^{4}(\mathbb{T})\right)}^{4} \leq\|u\|_{L^{5}\left(0, T ; W^{1,4}(\mathbb{T})\right)}^{4} \leq C .
$$

Since $L^{1}(\mathbb{T})$ embeddes continuously into $H^{-1}(\mathbb{T})$, the above estimates show that

$$
\begin{gathered}
\tau^{-1}\left\|u^{4}-\sigma_{\tau}\left(u^{4}\right)\right\|_{L^{5 / 4}\left(0, T ; H^{-3}(\mathbb{T})\right)} \leq 2\left\|u^{2}\left(u^{2}\right)_{x x x}-\frac{16}{3} u u_{x}^{3}\right\|_{L^{5 / 4}\left(0, T ; L^{2}(\mathbb{T})\right)} \\
+12\left\|\left(u^{2} u_{x}^{2}\right)_{x}\right\|_{L^{5 / 4}\left(0, T ; L^{2}(\mathbb{T})\right)}+8\left\|\left(u^{2}\right)_{x x}^{2}+\frac{16}{3} u_{x}^{4}\right\|_{L^{5 / 4}\left(0, T ; H^{-1}(\mathbb{T})\right)}
\end{gathered}
$$

is uniformly bounded.

LEMMA 3.5. The function $u^{(\tau)}$ satisfies

$$
\left\|\left(u^{(\tau)}\right)^{4}\right\|_{L^{5 / 4}\left(0, T ; H^{3}(\mathbb{T})\right)} \leq C .
$$

Proof. We set again $u=u^{(\tau)}$ and observe that

$$
\left(u^{4}\right)_{x x x}=24 u u_{x}^{3}+12 u^{2}\left(u_{x}^{2}\right)_{x}+2 u^{2}\left(u^{2}\right)_{x x x} .
$$

The first and last terms are uniformly bounded in $L^{5 / 4}\left(0, T ; L^{2}(\mathbb{T})\right)$, taking into account (3.18) and (3.19). Furthermore, the Hölder inequality with $p=8 / 3$ and $p^{\prime}=8 / 5$ and the bounds (3.15) and (3.17) imply that

$$
\begin{aligned}
\left\|u^{2}\left(u_{x}^{2}\right)_{x}\right\|_{L^{5 / 4}\left(0, T ; L^{2}(\mathbb{T})\right)}^{5 / 4} & \leq \int_{0}^{T}\|u(\cdot, t)\|_{L^{\infty}(\mathbb{T})}^{5 / 2}\left\|u_{x}(\cdot, t)^{2}\right\|_{H^{1}(\mathbb{T})}^{5 / 4} d t \\
& \leq\|u\|_{L^{20 / 3}\left(0, T ; L^{\infty}(\mathbb{T})\right)}^{5 / 2}\left\|u_{x}^{2}\right\|_{L^{2}\left(0, T ; H^{1}(\mathbb{T})\right)}^{5 / 4} \leq C .
\end{aligned}
$$

Hence, $\left(u^{4}\right)_{x x x}$ is uniformly bounded in $L^{5 / 4}\left(0, T ; L^{2}(\mathbb{T})\right)$. Furthermore, $u^{4}$ is uniformly bounded in $L^{5 / 4}\left(0, T ; L^{2}(\mathbb{T})\right)$. This gives the desired estimate.

3.3. The limit $\tau \rightarrow 0$. The a priori estimates of the previous subsection are sufficient to pass to the limit $\tau \rightarrow 0$. In fact, by Lemmas 3.4 and 3.5, we can apply the Aubin lemma (Theorem 5 in [23]) to obtain the existence of a subsequence of $\left(\left(u^{(\tau)}\right)^{4}\right)$, which is not relabeled, such that $\left(u^{(\tau)}\right)^{4} \rightarrow n$ strongly in $L^{5 / 4}\left(0, T ; W^{2, p}(\mathbb{T})\right)$ for all $p \leq \infty$ as $\tau \rightarrow 0$ for some limit function $n$. Here, we have employed the compact embedding of $H^{3}(\mathbb{T})$ into $W^{2, p}(\mathbb{T})$ for all $p \leq \infty$. In particular, $\left(u^{(\tau)}\right)$ converges 
pointwise a.e. Since $\left(u^{(\tau)}\right)^{4}$ is nonnegative, also the limit $n$ is nonnegative, and the function $u=\sqrt[4]{n}$ is well defined. Moreover, it holds

$$
\begin{aligned}
\tau^{-1}\left(\left(u^{(\tau)}\right)^{4}-\left(\sigma_{\tau} u^{(\tau)}\right)^{4}\right) \rightarrow\left(u^{4}\right)_{t} & \text { weakly in } L^{5 / 4}\left(0, T ; H^{-3}(\mathbb{T})\right) \\
\left(u^{(\tau)}\right)^{2} \rightarrow u^{2} & \text { weakly in } L^{2}\left(0, T ; H^{3}(\mathbb{T})\right)
\end{aligned}
$$

The strong convergence of $\left(u^{(\tau)}\right)^{4}$ to $u^{4}$ in $L^{5 / 4}\left(0, T ; L^{\infty}(\Omega)\right)$ and the inequality $\mid a-$ $\left.b\right|^{4} \leq\left|a^{4}-b^{4}\right|$ for all $a, b>0$ imply that

$$
\begin{aligned}
\left\|u^{(\tau)}-u\right\|_{L^{5}\left(0, T ; L^{\infty}(\mathbb{T})\right)}^{5} & \leq \int_{0}^{T}\left\|\left(u^{(\tau)}\right)^{4}-u^{4}\right\|_{L^{\infty}(\mathbb{T})}^{5 / 4} d t \\
& =\left\|\left(u^{(\tau)}\right)^{4}-u^{4}\right\|_{L^{5 / 4}\left(0, T ; L^{\infty}(\mathbb{T})\right)}^{5 / 4} \rightarrow 0,
\end{aligned}
$$

which means that

$$
u^{(\tau)} \rightarrow u \quad \text { strongly in } L^{5}\left(0, T ; L^{\infty}(\mathbb{T})\right)
$$

This result allows us to prove the strong convergence of $\left(u^{(\tau)}\right)^{2}$ to $u^{2}$ in $L^{2}(0, T$; $\left.H^{2}(\mathbb{T})\right)$. Indeed, the Gagliardo-Nirenberg inequality with $\theta=3 / 5$ and then the Hölder inequality give

$$
\begin{aligned}
\left\|\left(u^{(\tau)}\right)^{2}-u^{2}\right\|_{L^{2}\left(0, T ; H^{2}(\mathbb{T})\right)}^{2} & \leq C \int_{0}^{T}\left\|\left(u^{(\tau)}\right)^{2}-u^{2}\right\|_{H^{3}(\mathbb{T})}^{2 \theta}\left\|\left(u^{(\tau)}\right)^{2}-u^{2}\right\|_{L^{\infty}(\mathbb{T})}^{2(1-\theta)} d t \\
& \leq C\left\|\left(u^{(\tau)}\right)^{2}-u^{2}\right\|_{L^{2}\left(0, T ; H^{3}(\mathbb{T})\right)}^{2 \theta}\left\|\left(u^{(\tau)}\right)^{2}-u^{2}\right\|_{L^{2}\left(0, T ; L^{\infty}(\mathbb{T})\right)}^{2(1-\theta)}
\end{aligned}
$$

By (3.14), first factor is bounded, whereas the second factor converges to zero, by the above result. Thus,

$$
\left(u^{(\tau)}\right)^{2} \rightarrow u^{2} \quad \text { strongly in } L^{2}\left(0, T ; H^{2}(\mathbb{T})\right) .
$$

As a consequence, we infer that

$$
\begin{array}{r}
\left(u^{(\tau)}\right)^{2}\left(u_{x}^{(\tau)}\right)^{2}=\frac{1}{4}\left[\left(u^{(\tau)}\right)^{2}\right]_{x}^{2} \rightarrow \frac{1}{4}\left(u^{2}\right)_{x}^{2}=u^{2} u_{x}^{2} \quad \text { strongly in } L^{1}\left(0, T ; L^{1}(\mathbb{T})\right), \\
{\left[\left(u^{(\tau)}\right)^{2}\right]_{x x}^{2} \rightarrow\left(u^{2}\right)_{x x}^{2} \quad \text { strongly in } L^{1}\left(0, T ; L^{1}(\mathbb{T})\right) .}
\end{array}
$$

Taking into account the uniform bounds (3.20) and (3.21), it follows that

$$
\begin{gathered}
\left(u^{(\tau)}\right)^{2}\left(u_{x}^{(\tau)}\right)^{2} \rightarrow u^{2} u_{x}^{2} \quad \text { weakly in } L^{5 / 4}\left(0, T ; L^{2}(\mathbb{T})\right), \\
{\left[\left(u^{(\tau)}\right)^{2}\right]_{x x}^{2} \rightarrow\left(u^{2}\right)_{x x}^{2} \quad \text { weakly in } L^{5 / 4}\left(0, T ; L^{2}(\mathbb{T})\right) .}
\end{gathered}
$$

In view of the estimate (3.20), we obtain further

$$
\left(\left(u^{(\tau)}\right)^{2}\left(u_{x}^{(\tau)}\right)^{2}\right)_{x} \rightarrow\left(u^{2} u_{x}^{2}\right)_{x} \quad \text { weakly in } L^{5 / 4}\left(0, T ; L^{2}(\mathbb{T})\right) .
$$

The strong convergence of $u^{(\tau)}$ (see (3.25)) and the weak convergence of the sequence $\left[\left(u^{(\tau)}\right)^{2}\right]_{x x x}$ (see $\left.(3.24)\right)$ imply that $\left(u^{(\tau)}\right)^{2}\left[\left(u^{(\tau)}\right)^{2}\right]_{x x x}$ converges weakly to $u^{2}\left(u^{2}\right)_{x x x}$ in $L^{10 / 7}\left(0, T ; L^{2}(\mathbb{T})\right)$. In particular,

$$
\left(u^{(\tau)}\right)^{2}\left[\left(u^{(\tau)}\right)^{2}\right]_{x x x} \rightarrow u^{2}\left(u^{2}\right)_{x x x} \quad \text { weakly in } L^{5 / 4}\left(0, T ; L^{2}(\mathbb{T})\right) .
$$


By Proposition 6.1, applied to $\alpha=2, \beta=2 / 3, \gamma=4 / 3$ and $\gamma=1$, respectively, we obtain

$$
u^{(\tau)}\left(u_{x}^{(\tau)}\right)^{3}=\left(\frac{3}{4}\right)^{3}\left[\left(u^{(\tau)}\right)^{4 / 3}\right]_{x}^{3} \rightarrow\left(\frac{3}{4}\right)^{3}\left(u^{4 / 3}\right)_{x}^{3}=u u_{x}^{3}
$$

weakly in $L^{10 / 7}\left(0, T ; L^{2}(\mathbb{T})\right)$ and

$$
\left(u_{x}^{(\tau)}\right)^{4} \rightarrow u_{x}^{4} \quad \text { weakly in } L^{3 / 2}\left(0, T ; L^{3 / 2}(\mathbb{T})\right),
$$

since $\left(u^{(\tau)}\right)$ is bounded in $L^{5}\left(0, T ; L^{\infty}(\mathbb{T})\right)$ and $L^{6}\left(0, T ; W^{1,6}(\mathbb{T})\right)$.

In view of the convergence results (3.23), (3.27)-(3.31), we can perform the limit $\tau \rightarrow 0$ in (3.13) to conclude that $u$ solves

$$
\left(u^{4}\right)_{t}=2\left(u^{2}\left(u^{2}\right)_{x x x}-\frac{16}{3} u u_{x}^{3}\right)_{x x x}-12\left(u^{2} u_{x}^{2}\right)^{(\mathrm{iv})}+8\left(\left(u^{2}\right)_{x x}^{2}+\frac{16}{3} u_{x}^{4}\right)_{x x}
$$

in the sense of $L^{5 / 4}\left(0, T ; H^{-3}(\mathbb{T})\right.$. This proves Theorem 1.1.

4. Exponential time decay of the solutions. We prove Theorem 1.2. Let $k \in \mathbb{N}, \tau>0$, and $t \in((k-1) \tau, k \tau]$. Furthermore, let $u_{k}$ be the periodic solution to (3.1) constructed in Lemma 3.1. Then, by the proof of Lemma 3.1, using the inequality of Lemma 2.3 instead of Lemma 2.1,

$$
E\left(u_{k}^{4}\right)+\tau \kappa_{2} \int_{\mathbb{T}}\left(u_{k}^{2}\right)_{x x x}^{2} d x \leq E\left(u_{k-1}^{4}\right), \quad k \in \mathbb{N},
$$

where $\kappa_{2}$ is given in (1.8). We employ the generalized logarithmic Sobolev inequality with optimal constant (see Corollary 4.3 in [12]):

$$
\int_{\mathbb{T}} v^{2} \log \left(\frac{v^{2}}{\left\|v^{2}\right\|_{L^{1}(\mathbb{T})}}\right) d x \leq \frac{2}{(2 \pi)^{6}} \int_{\mathbb{T}} v_{x x x}^{2} d x, \quad v \in H^{3}(\mathbb{T}),
$$

applied to $v=u_{k}^{2}$. Then, since $u_{0}^{4}$ is assumed to have unit mass, $\left\|u_{k}^{4}\right\|_{L^{1}(\mathbb{T})}=$ $\left\|u_{0}^{4}\right\|_{L^{1}(\mathbb{T})}=1$

$$
E\left(u_{k}^{4}\right) \leq \frac{2}{(2 \pi)^{6}} \int_{\mathbb{T}}\left(u_{k}^{2}\right)_{x x x}^{2} d x
$$

and the above entropy inequality becomes

$$
E\left(u_{k}^{4}\right)+\frac{1}{2} \tau \kappa_{2}(2 \pi)^{6} E\left(u_{k}^{4}\right) \leq E\left(u_{k-1}^{4}\right), \quad k \in \mathbb{N} .
$$

This recursive inequality can be solved:

$$
E\left(u_{k}^{4}\right) \leq E\left(u_{0}^{4}\right)\left(1+\frac{1}{2} \tau \kappa_{2}(2 \pi)^{6}\right)^{-k}, \quad k \in \mathbb{N}
$$

For $t \in((k-1) \tau, k \tau]$, we can write this inequality as

$$
E\left(\left(u^{(\tau)}\right)^{4}\right) \leq E\left(u_{0}^{4}\right)\left(1+\frac{1}{2} \tau \kappa_{2}(2 \pi)^{6}\right)^{-t / \tau}
$$

Since $u^{(\tau)}(x, t)$ converges a.e. to $u(x, t)$ as $\tau \rightarrow 0$ and $\left(1+\frac{1}{2} \tau \kappa_{2}(2 \pi)^{6}\right)^{-t / \tau}$ converges to $\exp \left(-\frac{1}{2} \tau \kappa_{2}(2 \pi)^{6} t\right)$, we can perform the limit $\tau \rightarrow 0$ in the above inequality which implies the assertion. 


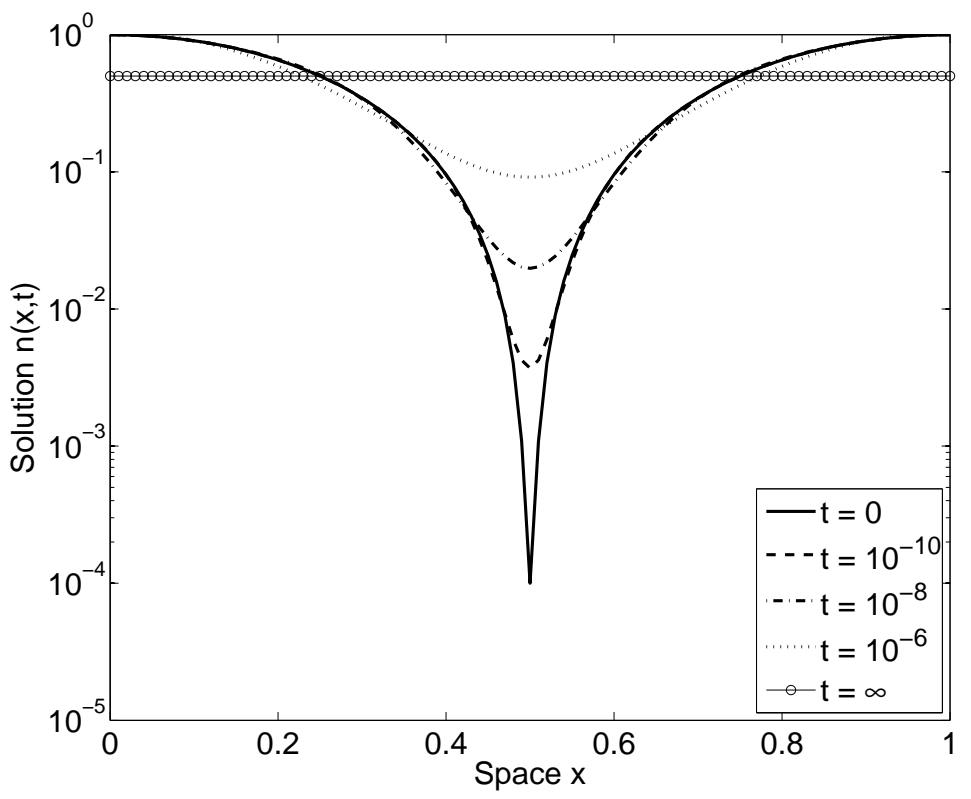

FIG. 5.1. Time evolution of the solution $n(x, t)$ with initial condition (5.1) with $m=1$.

5. Numerical examples. The numerical examples of this section illustrate the long-time behavior of the solution to (1.1). They also underline the preservation of nonnegativity and the nonmonotonic behavior of the solutions. For the numerical experiments we choose, as in [3, 18], the initial datum

$$
n_{0}(x)=\varepsilon+\cos ^{2 m}(\pi x), \quad x \in(0,1),
$$

where $\varepsilon>0$ is a small parameter. We take $\varepsilon=10^{-4}$ and $m=1$ or $m=8$. For the computations, we discretize (1.1) in time by the implicit Euler scheme and in space by central finite differences. The discrete nonlinear system is solved by the Newton method; no damping was necessary. The initial guess is chosen as the solution of the previous time level. The time step is $\Delta t=10^{-10}$, and we used 100 grid points.

Figures 5.1 and 5.2 show the evolution of the initial datum with $m=1$ and $m=8$, respectively. Notice that we use a logarithmic scale for the ordinate. The solution for $m=1$ increases rapidly until it reaches the constant steady state $n_{\text {inf }}=$ $1 / 2+\varepsilon$. On the other hand, the solution for $m=8$ starts with one higher-order minimum, bifurcates into two local minima and reduces to one extremum again, before converging to the constant steady state $n_{\text {inf }}=6435 / 32768+\varepsilon \approx 0.196$. In particular, the evolution is not monotone. This behavior is similar to that of the fourth-order equation $(1.3)[3,18]$.

The difference $n(\cdot, t)-n_{\text {inf }}$ in the $L^{1}$ norm versus time $t$ is presented in Figures 5.3 and 5.4. In both cases, we see that the decay of the difference is approximately exponential confirming the analytical result of Theorem 1.2.

6. Appendix. We prove the following result which is a consequence of Theorem 5.4 .4 of $[1]$. 


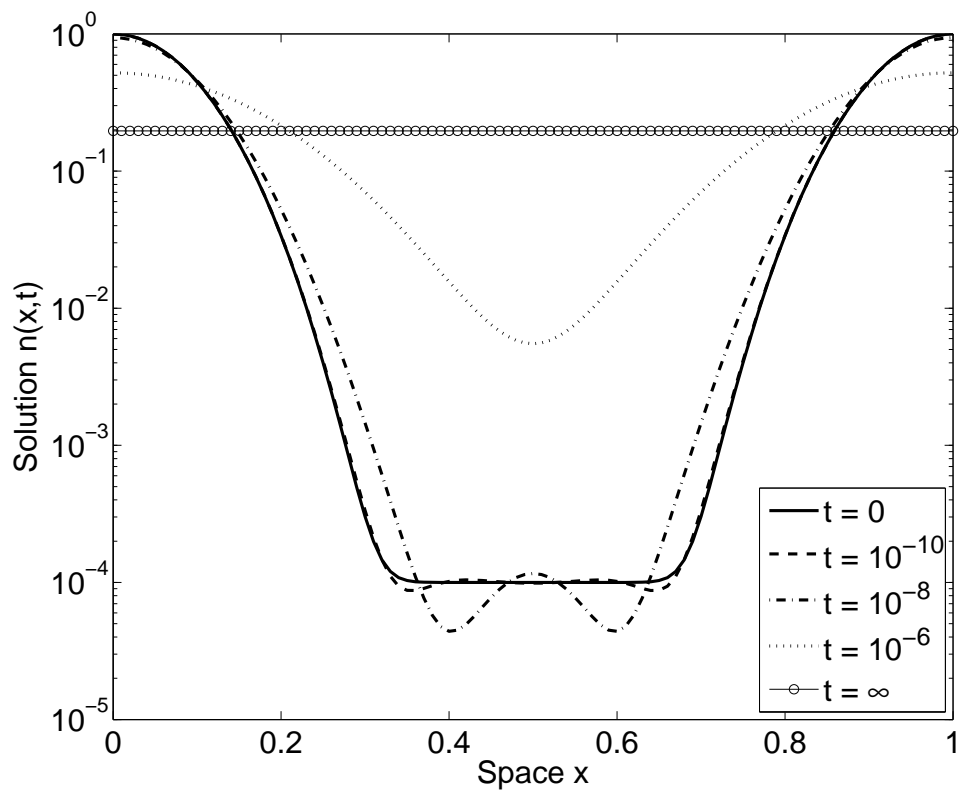

FIG. 5.2. Time evolution of the solution $n(x, t)$ with initial condition (5.1) with $m=8$.

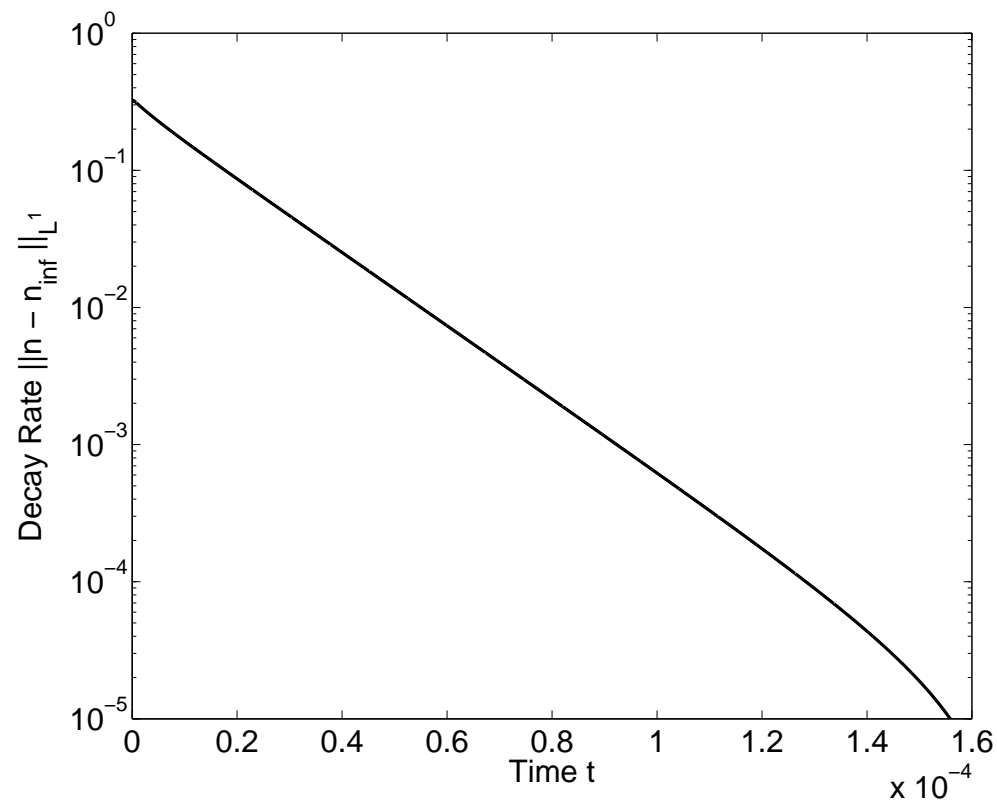

FIG. 5.3. Decay of the difference $\left\|n(\cdot, t)-n_{\mathrm{inf}}\right\|_{L^{1}(0,1)}$ using the initial condition (5.1) with $m=1$. 


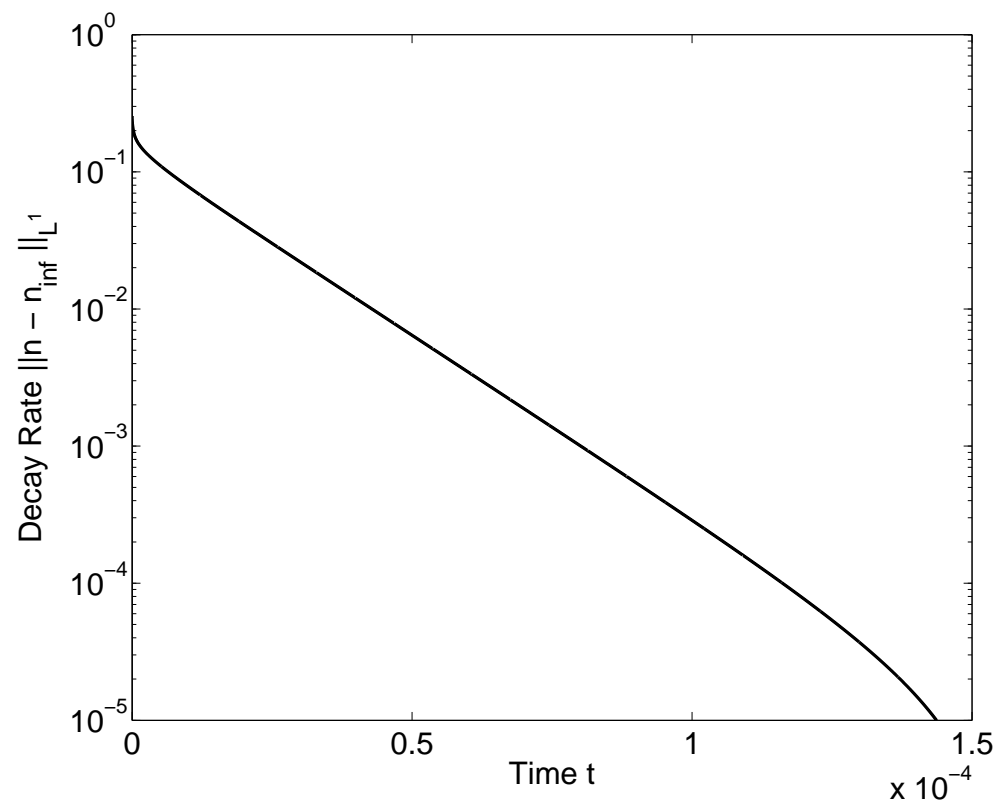
$m=8$.

FIG. 5.4. Decay of the difference $\left\|n(\cdot, t)-n_{\text {inf }}\right\|_{L^{1}(0,1)}$ using the initial condition (5.1) with

Proposition 6.1. Let $\Omega \subset \mathbb{R}^{d}$ be a bounded domain $(d \geq 1), 0<\beta<\gamma<\alpha<$ $\infty, 1<p, q, r<\infty$ with $\alpha p=\beta q=\gamma r$, and let $\left(u_{\varepsilon}\right)$ be a sequence of strictly positive functions on $\Omega$ with the following properties:

$$
\begin{aligned}
& \text { (i) } u_{\varepsilon}^{\alpha} \rightarrow u^{\alpha} \text { strongly in } W^{1, p}(\Omega) \text {, as } \varepsilon \rightarrow 0 \text {, } \\
& \text { (ii) }\left(u_{\varepsilon}^{\beta}\right) \text { bounded in } W^{1, q}(\Omega) \text {. }
\end{aligned}
$$

Then $u_{\varepsilon}^{\gamma} \rightarrow u^{\gamma}$ strongly in $W^{1, r}(\Omega)$ and, in particular,

$$
\left|\nabla\left(u_{\varepsilon}^{\gamma}\right)\right|^{r} \rightarrow\left|\nabla\left(u^{\gamma}\right)\right|^{r} \quad \text { strongly in } L^{1}(\Omega) .
$$

Furthermore, when $\left(u_{\varepsilon}\right)$ is a sequence of strictly positive functions on $\Omega \times(0, T)$, where $T>0$, and (i) and (ii) hold with $W^{1, s}(\Omega)$ replaced by $L^{s}\left(0, T ; W^{1, s}(\Omega)\right)$, where $s=p$ and $s=q$, respectively, then

$$
\left|\nabla\left(u_{\varepsilon}^{\gamma}\right)\right|^{r} \rightarrow\left|\nabla\left(u^{\gamma}\right)\right|^{r} \quad \text { weakly in } L^{1}\left(0, T ; L^{1}(\Omega)\right) .
$$

Proof. Let $\mu_{\varepsilon}, \mu$ be the measures corresponding to the densities $u_{\varepsilon}^{c}, u^{c}$, respectively, where $c=\alpha p$. Then, as $\left(u_{\varepsilon}^{c}\right)$ converges to $u^{c}$ strongly in $L^{1}(\Omega),\left(\mu_{\varepsilon}\right)$ converges narrowly to $\mu$. The functions $v_{\varepsilon}=\nabla u_{\varepsilon} / u_{\varepsilon}$ belong to the space $L^{p}\left(\mu_{\varepsilon} ; \Omega\right)$ since

$$
\left\|v_{\varepsilon}\right\|_{L^{p}\left(\mu_{\varepsilon} ; \Omega\right)}^{p}=\int_{\Omega}\left|v_{\varepsilon}\right|^{p} d \mu_{\varepsilon}=\int_{\Omega}\left|v_{\varepsilon}\right|^{p} u_{\varepsilon}^{c} d x=\alpha^{-p} \int_{\Omega}\left|\nabla u_{\varepsilon}^{\alpha}\right|^{p} d x<\infty .
$$

Thus, assumption (i) implies that $\left(v_{\varepsilon}\right)$ converges strongly to $v=\nabla u / u \in L^{p}(\mu ; \Omega)$ in the sense of Definition 5.4.3 of [1]. By Theorem 5.4.4 (iii) of [1], the transport plans 
$\gamma_{\varepsilon}=\left(\mathrm{id} \times v_{\varepsilon}\right)_{\#} \mu_{\varepsilon}$ converge narrowly to $\gamma=(\mathrm{id} \times v)_{\#} \mu$. Here, id denotes the identity function on $\Omega$ and $f_{\#} \mu$ is the push-forward of the measure $\mu$ through the function $f$. Since $\left(\mu_{\varepsilon}\right)$ has uniformly integrable $p$-moments,

$$
\sup _{\varepsilon>0} \int_{\Omega}|y|^{p} d \gamma_{\varepsilon}(x, y)=\sup _{\varepsilon>0} \int_{\Omega}\left|v_{\varepsilon}(x)\right|^{p} d \mu_{\varepsilon}(x)<\infty,
$$

we infer from the same theorem that, as $\varepsilon \rightarrow 0$,

$$
\int_{\Omega} f\left(x, v_{\varepsilon}(x)\right) d \mu_{\varepsilon}(x) \rightarrow \int_{\Omega} f(x, v(x)) d \mu
$$

for all functions $f$ with at most $p$-growth. In view of assumption (ii), this convergence also holds for all functions with at most $r$-growth, $r<q$ (see Lemma 5.1.7 of [1]). Choosing $f(x, y)=|y|^{r}$ yields

$$
\int_{\Omega}\left|\nabla\left(u_{\varepsilon}^{\gamma}\right)\right|^{r} d x=\gamma^{r} \int_{\Omega}\left|v_{\varepsilon}\right|^{r} d \mu_{\varepsilon} \rightarrow \gamma^{r} \int_{\Omega}|v|^{r} d \mu=\int_{\Omega}\left|\nabla\left(u^{\gamma}\right)\right|^{r} d x .
$$

Thus, $\left(u_{\varepsilon}^{\gamma}\right)$ converges to $u^{\gamma}$ in norm and weakly in $W^{1, r}(\Omega)$, which implies strong convergence (see Prop. 21.23 (g) of [26]).

In the case of the spaces $L^{s}\left(0, T ; W^{1, s}(\Omega)\right), s=p, q, r$, the above arguments leading to (6.1) still hold, where now $x \in \Omega \times(0, T)$. Then, choosing $f(x, y)=\phi(x)|y|^{r}$ with $\phi \in L^{\infty}\left(0, T ; L^{\infty}(\Omega)\right)$ gives the weak convergence of $\left|\nabla\left(u_{\varepsilon}^{\gamma}\right)\right|^{r}$ to $\left|\nabla\left(u^{\gamma}\right)\right|^{r}$ in $L^{1}\left(0, T ; L^{1}(\Omega)\right)$.

\section{REFERENCES}

[1] L. Ambrosio, N. Gigli, and G. Savaré. Gradient Flows in Metric Spaces and in the Space of Probability Measures. Birkhäuser, Basel, 2005.

[2] F. Bernis and A. Friedman. Higher order nonlinear degenerate parabolic equations. J. Diff. Eqs. 83 (1990), 179-206.

[3] P. Bleher, J. Lebowitz, and E. Speer. Existence and positivity of solutions of a fourth-order nonlinear PDE describing interface fluctuations. Commun. Pure Appl. Math. 47 (1994), 923-942.

[4] M. Burger. Numerical simulation of anisotropic surface diffusion with curvature-dependent energy. J. Comput. Phys. 203 (2005), 602-625.

[5] G. Caginalp and M. Burger. Higher-order phase field models and detailed anisotropy. Phys. Rev. B 34 (1986), 4940-4943.

[6] J. A. Carrillo, A. Jüngel, P. Markowich, G. Toscani, and A. Unterreiter. Entropy dissipation methods for degenerate parabolic problems and generalized Sobolev inequalities. Monatsh. Math. 133 (2001), 1-82.

[7] J. A. Carrillo, A. Jüngel, and S. Tang. Positive entropic schemes for a nonlinear fourth-order equation. Discrete Contin. Dynam. Sys. B 3 (2003), 1-20.

[8] P. Constantin, T. Dupont, R. Goldstein, L. Kadanoff, M. Shelley, and S.-M. Zhou. Droplet breakup in a model of the Hele-Shaw cell. Phys. Rev. E 47 (1993), 4169-4181.

[9] R. Dal Passo and H. Garcke. Solutions of a fourth order degenerate parabolic equation with weak initial trace. Ann. Scuola Norm. Sup. Pisa 28 (1999), 153-181.

[10] P. Degond, F. Méhats, and C. Ringhofer. Quantum energy-transport and drift-diffusion models. J. Stat. Phys. 118 (2005), 625-665.

[11] B. Derrida, J. Lebowitz, E. Speer, and H. Spohn. Fluctuations of a stationary nonequilibrium interface. Phys. Rev. Lett. 67 (1991), 165-168.

[12] J. Dolbeault, I. Gentil, and A. Jüngel. A logarithmic fourth-order parabolic equation and related logarithmic Sobolev inequalities. Commun. Math. Sci. 4 (2006), 275-290.

[13] J. Evans, V. Galaktionov, and J. King. Unstable sixth-order thin film equation: I. Blow-up similarity solutions. Nonlinearity 20 (2007), 1799-1841.

[14] H. Greenspan. On the motion of a small viscous droplet that wets a surface. J. Fluid Mech. 84 (1978), 125-143. 
[15] A. Jüngel and D. Matthes. An algorithmic construction of entropies in higher-order nonlinear PDEs. Nonlinearity 19 (2006), 633-659.

[16] A. Jüngel and D. Matthes. The Derrida-Lebowitz-Speer-Spohn equation: existence, nonuniqueness, and decay rates of the solutions. SIAM J. Math. Anal. 39 (2008), 1996-2015.

[17] A. Jüngel and D. Matthes. A review on results for the Derrida-Lebowitz-Speer-Spohn equation. To appear in Proccedings of the EQUADIFF07 Conference, 2009.

[18] A. Jüngel and R. Pinnau. Global non-negative solutions of a nonlinear fourth-order parabolic equation for quantum systems. SIAM J. Math. Anal. 32 (2000), 760-777.

[19] J. King. The isolation oxidation of silicon: the reaction-controlled case. SIAM J. Appl. Math. 49 (2000), 10641080.

[20] J. King. Two generalisations of the thin film equation. Math. Comput. Modelling 34 (2001), 737-756.

[21] P.-L. Lions and C. Villani. Régularité optimale de racines carrées. C. R. Acad. Sci. Paris, Sér. I 321 (1995), 1537-1541.

[22] M. Short. A parabolic linear evolution equation for cellular detonation instability. Combustion Theory Modeling 1 (1997), 313-346.

[23] J. Simon. Compact sets in the space $L^{p}(0, T ; B)$. Ann. Mat. Pura Appl. 146 (1987), 6596.

[24] W. Smith, S. Howison, and D. Mayers. Numerical and asymptotic solution of a sixth-order nonlinear diffusion equation and related coupled systems. IMA J. Appl. Math. 57 (1996), 79-98.

[25] M. Taylor. Partial Differential Equations. III. Nonlinear Equations. Springer, New York, 1997.

[26] E. Zeidler. Nonlinear Functional Analysis and its Applications. Springer, Berlin, 1990. 